\title{
High-dose intermittent sunitinib therapy retains the crown
}

The approved first-line therapy for advanced-stage renal cell carcinoma (RCC) is high-dose ( $50 \mathrm{mg}$ per day) intermittent dosing of sunitinib ( 4 weeks on and 2 weeks off treatment, called schedule 4/2). Although a low-dose (37.5 mg per day) continuous daily dosing (CDD) of sunitinib has been recommended as an alternative schedule, a new study now suggests that the low-dose CDD schedule has no benefit in efficacy and safety over the approved high-dose schedule.

In this multicentre randomized phase II trial, 292 patients with advanced-stage RCC were randomly assigned to receive either of the two sunitinib dosing schedulesthe intermittent dosing group received sunitinib for a median of 5 months and those in the CDD group were treated for a median of 6 months. The primary end point was time to progression (TTP), and the secondary end points included objective response rate (ORR), overall survival and safety. A longer median TTP (9.9 months) was reported in the schedule $4 / 2$ group than in the CDD group (7.1 months).

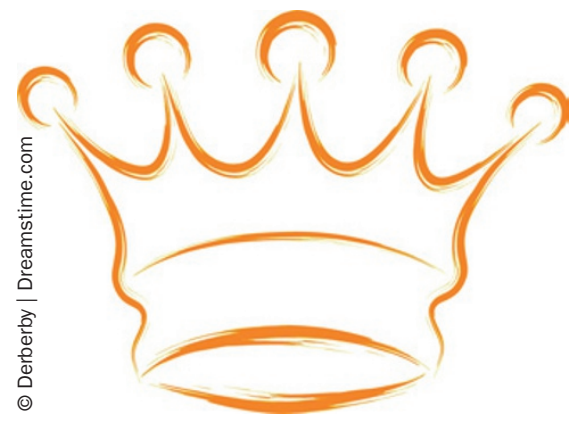

Median progression-free survival (PFS) was also increased in the schedule $4 / 2$ arm (8.5 months versus 7 months). A similar trend was reported for ORR (32\% versus $28 \%$ ), whereas no difference was reported in overall survival between the two arms (23.1 months versus 23.5 months).

Safety analyses showed a reduced risk of tumour progression among patients in the schedule 4/2 arm compared with patients in the CDD group. Moreover, a similar number of adverse events, such as fatigue, hypertension and diarrhoea, were noted in the two schedule groups. In addition, fewer patients stopped sunitinib therapy in the schedule $4 / 2$ arm (11\%) than those in the CDD arm (15\%).

Patient-reported outcome data were also compared between the two dosing schedules. The scoring pattern reflected a reversible on and off effect of schedule $4 / 2$ on patients' quality of life, whereas a stable effect was suggested among those in the CDD group.

Overall, this study demonstrates that low-dose continuous dosing of sunitinib has no benefit in efficacy and safety over the approved high-dose intermittent schedule. If anything, the increased TTP and PFS, as well as a trend for a reversible effect on quality of life with schedule 4/2 suggest a favourable outcome with this regimen and support its use as the approved first-line therapy of advanced-stage RCC.

Iley Ozerlat

Original article Motzer, R. J. et al. Randomized phase II trial of sunitinib on an intermittent versus continuous dosing schedule as first-line therapy for advanced renal cell carcinoma. J. Clin. Oncol. doi:10.1200/JC0.2011.36.4133 